\title{
Long-term effects from individualized and continuously optimized physiotherapy in cystic fibrosis
}

\author{
Lannefors $L^{*}$, Philipsen $L$ and Pressler T \\ Copenhagen CF centre, University Hospital Rigshospitalet, Denmark
}

\begin{abstract}
Introduction: Modern physiotherapy in Cystic Fibrosis (CF) includes airway clearance (AC) as well as inhalation therapy and physical exercise. Many studies have reported different $\mathrm{AC}$ techniques equally effective, but not in all $\mathrm{CF}$ patients. Patient preference is important for adherence and thereby for the result. The pulmonary disease progresses and life changes. What effects can continuously individually optimized physiotherapy have in CF?

Method: In this interventional long-term pilot study thirteen adult CF patients (followed for 1 year) learnt to utilize different AC techniques and to choose technique(s) for everyday use, achieving a certain physiologic strategy. They saw a physiotherapist for optimization and education every month, were guided in inhalation therapy and physical exercise, and were contacted once a week to optimize and keep up with their treatment programs. Patients were their own controls as the analysing of results were compared with the two immediate annual years pre-existing clinical and laboratory data collected as part of the patients' regular medical care.
\end{abstract}

Results: Mean $\mathrm{FEV}_{1}$ and FVC change rate changed from annual decline the two prior years to improvement after the intervention year. Chest mobility increased significantly. Peak working capacity, oxygen saturation, active hours during day and quality of life improved slightly.

Conclusion: Patient cooperation, individualizing and continuously optimizing flexible physiotherapy based on needs and patients' preference show good long-term physical effect and quality of life. Further and longer studies on effects on early cooperation between physiotherapist and patient are necessary.

\section{Introduction}

In Cystic Fibrosis (CF) the mucus obstruction in pulmonary airways is originated by dehydration of airway surface liquid and impaired mucociliary clearance, commencing in the small airways at the start of life [1]. Respiratory physiotherapy is considered an important part of $\mathrm{CF}$ care, but used to comprise mainly airway clearance (AC) therapy and not necessarily aiming at the most peripheral airways [2-4]. Modern respiratory physiotherapy includes inhalation therapy, AC therapy and physical activity/exercise, all aiming at reducing disease progression and avoiding complications rather than rehabilitating [3-7].

The prevailing AC technique used to be postural drainage (PD) with percussion and vibration, utilized in hospital as well as in everyday care at home. It was time-consuming and not possible for patients to accomplish themselves, they were dependent on a parent/ assistant to have it done properly. But, since late 1970:es different AC techniques have been developed and evaluated [3-7]. Different AC techniques are based upon different respiratory physiology theories that may be important for the single patient, dependent on to what extent the pulmonary airways and lung tissue is damaged by the disease [3]. Sadly, publications often define the physiology of the studied AC techniques poorly, if at all, sometimes also the patient instructions are unsatisfactory described, and the study cannot be repeated. Most studies, irrespective of lasting for a single treatment or up to three years, report different techniques equally effective, although not in all patients $[4,5]$. However, long term studies confirm that individual patient preference is an important factor for adherence and thereby for the result $[4,5]$. Patient preference may vary as well as lung function change over time.
Inhalation therapy is continuously expanding as the therapeutic targets increase [8]. Due to the different aim that the different kinds of drugs have (bronchodilator, mucolytic, anti-inflammatory or antimicroorganic) the treatment strategy is of great importance due to the expected deposition pattern and amounts of mucus expectorated during the AC therapy [3]. We also know increasingly more about what effects optimal aerosol quality and good inhalation technique have on deposition pattern, especially in patients with an influenced respiratory physiology and affected breathing pattern $[3,9]$.

Physical activity has been recommended a part of every-day life since long, and to an increasing event physical exercise is becoming part of basic care $[3,6,7,10-14]$. Physical capacity test is reported a sensitive measure of early changes in pulmonary disease and is recommended to be performed regularly [14]. Studies, old and new, report improved physical capacity from supervised physical exercise programmes over short term, but status soon returned to basic when supervision ceased [15-17]. However, some studies with less supervised physical exercise as part of the basic treatment showed improvement of physical capacity and stabilized lung function $[6,7,10,11]$. Focusing on individualizing the physical exercise, favouring adherence by integrating it with patients' lifestyle and desires gave positive long-term effect $[6,7,10,12,13]$.

*Correspondence to: Lannefors L, Gothenburg CF-centre, Gröna Stråket 9, plan 5, Sahlgrenska University Hospital, 41345 Göteborg, Sweden, E-mail: llannefors@gmail.com

Key words: cystic fibrosis, airway clearance, physical exercise, physiotherapy

Received: June 28, 2018; Accepted: July 20, 2018; Published: July 25, 2018 
The objective of this study was to evaluate long-term effects of modern physiotherapy including inhalation therapy, AC therapy and physical exercise, when continually optimized together with the patient to fit the individual the best during every-day life for a year.

\section{Methods}

\section{Study design}

This is an interventional long-term pilot study.

\section{Patients}

Adult CF patients treated at Copenhagen CF centre were recruited. CF diagnosis was based on sweat chloride testing, confirmed by the finding of two disease causing mutations. Data were obtained from the clinical CF database, holding prospectively entered patient data. Thus, the analyses were based on pre-existing clinical and laboratory data collected as part of the patients' regular medical care. Patients were their own controls. The data used for comparison of effect were the results from the routine annual tests from the two immediate years prior to the intervention. Only those adult patients who were not accustomed to regular physiotherapy contact and evaluation, and not used to physical exercise as a part of the basic treatment were invited to participate. Intervention duration was one year.

Inclusion criteria: $>18$-year-old and forced expiratory volume in one second $\left(\mathrm{FEV}_{1}\right)>40$ percent predicted (\% pred). Exclusion criteria: pain, handicap and participation in another study.

A physician not involved in the study saw the patients at their traditional scheduled monthly controls to the CF clinic. By routine these controls always included lung function test and general checkup. The general CF treatment was continued and/or changed, handled following a treatment pattern since many years for all patients, based on needs defined by the physician and agreed upon with the patient.

\section{Intervention Physiotherapy}

During routine monthly controls at the out-patient clinic the included patients met the physiotherapist for evaluation and optimizing or changing the treatment when needed or desired, practicing therapy and making new agreements. In-between, patients were contacted weekly via telephone or mail to support and maintain motivation. Content and technique(s) were possible to adapt to individual needs and desires at each contact. The different treatment quantities could be both increasing and decreasing at times during the study depending on need, but always striving for being optimal and acceptable for the patient, fitting into their every-day life to facilitate adherence. Visiting the physiotherapist during the intervention followed a certain strategy, in collaboration with the patient;

- evaluate and settle AC therapy

- settle inhalation therapy and strategy, if prescribed

- tailor and agree on a physical exercise programme

- individualize treatment education at contacts

- change/optimize parts of physiotherapy if/when needed or desired

\section{Inhalation therapy}

A physician, not involved in the study, was responsible for choice and prescription of inhalation therapy. Drug(s) included could be; broncho-dilator administered as a powder or solution to start with

- hypertonic saline 5-7\%, inhalation alternated with AC therapy or performed simultaneously

- RhDNase most often after AC therapy

antibiotic administered as powder or solution at the end

The physiotherapist was responsible for the choice of device when adequate, teaching inhalation technique and establishing an optimal treatment strategy.

\section{Airway clearance therapy}

All AC therapy was based on the same respiratory physiology "treatment cycle", consisting of 4 steps;

1) open airways,

2) mobilize,

3) transport and

4) evacuate secretions

Each treatment session could comprise a single, specific AC technique, or the "treatment cycle" consisting of parts of techniques presented in the International Physiotherapy Group for CF booklet [3]. The treatment was performed in sitting only or including side lying. There was no minimum time of AC. A session included the "treatment cycle" as many times needed and/or accepted by the patient. The choice of technique(s) was based on the effects achieved and on patient preferences, which may have differed during the study intervention. Deep inspirations, recruiting closed airways, always ended each AC therapy session. The patients were taught to do airway clearance twice a day to get a feeling of how much mucus there was and to clear the mucus they had. The airway clearance could be five minutes or more, but currently always noticing and working with the mucus they had, and how much they had varied during the intervention. Decreasing adherence, patient dissatisfaction with the treatment as well as lung function impairment were reasons for optimizing and/or changing AC techniques at each physiotherapy contact, all dependent on agreements and needs.

\section{Physical exercise}

Each patient performed unsupervised physical exercise $\geq 3$ times per week. Agreement was made with the patient regarding the physical exercise programme content;

- endurance training, preferably both aerobic and anaerobic, based on

- weight bearing exercises

- mobility training, primarily focusing on chest wall and auxiliary muscles

- muscle strengthening exercises, primarily comprising postural muscles

Each patient's potential physical exercise/sport at study start could be optimized or intensified and supplemented with exercises representing the aims, or new programme(s) were tailored. During the study intervention, the physical exercise programme could be changed depending on patient preferences, and the listed aims. 


\section{Outcomes}

\section{Parameters}

The parameters utilized were

- forced expiratory volume in one second $\left(\mathrm{FEV}_{1}\right)$

- forced vital capacity (FVC)

- functional residual capacity (FRC)

- lung clearance index (LCI)

- chest mobility

- peak working capacity (PWC) with transcutaneous oxygen saturation $(\mathrm{SpO} 2)$, heart rate and work of breathing

- body mass index (BMI)

- quality of life

- hours of physical activity during every-day life

\section{Details}

$\mathrm{FEV}_{1}, \mathrm{FVC}$ and BMI from the last two annual tests prior to the intervention served as control and were collected from patient records. $\mathrm{FEV}_{1}$ and FVC were performed standardised by a biomedical analytic, following the routine at the centre, in accordance with the ATS/ERS guidelines using Jaeger Master Screen Pro ${ }^{\circledR}$ (CareFusion, Hoechberg, Germany). Lung function is expressed as mean \% pred of the Global Lung Function Initiative (GLI) 2012 [18]. LCI (units) and FRC (litres) were measured by multiple breath washout inspiring $100 \%$ oxygen while nitrogen was washed out of the airways (Exhalyzer $D^{\oplus}$, IntraMedic $A B$, Gentofte, Denmark), following test routines [19]. Three measurements were performed at each time, from where a mean was calculated.

Peak Working Capacity (PWC) was performed as a ramp test on an electrically braked ergometer bicycle (Technogym ${ }^{\circledR}$, Pedan, Køge, Denmark), the Godfrey ramp test protocol was utilized [20]. Patients were instructed to keep the cadence to $60 / \mathrm{min}$. The PWC is expressed as \%pred Nordenfeldt [21]. Patients were requested to judge the work of breathing with the Borg CR10 scale [22]. With the help of a forehead electrode the $\mathrm{SpO} 2$ and heart rate was registered prior to, each minute during and three minutes after the test. The test was finished by routine when patients did not manage to attain the pre-defined cadence, or they indicated exhaustiveness.

Chest mobility was measured at rest as the difference in centimetre circumference between maximal inspiration and maximal expiration with a measuring tape around the chest at the Sternum Processus Xiphoideus. Patients were lying supine horizontal, hips bowed $45^{\circ}$ and knees $90^{\circ}$, and arms stretched along the body sides.

Quality of life was measured with the Cystic Fibrosis QuestionnaireRevised (CFQ-R) for adults and the Physical, Emotional, Health perception and Respiratory domains were utilized [23]. Hours of physical activity was measured with a Physical Activity Scale (PAS), which is a subjective measure where patients report how many hours during a normal 24-hour day they are physically active in different intensities. The scale has been validated in Denmark to measure physical activity in different patient groups and in healthy people [24].

\section{Data analysis}

$\mathrm{FEV}_{1}$ \%pred, FVC \%pred and BMI are referring to mean annual individual data from the 2 years control period immediately prior to the study, the study start and after one year of intervention. LCI, FRC, chest mobility, PWC \%pred including SpO2, quality of life and hours of physical activity during every-day life refer to individual data from study start and end of the intervention year. Data are expressed as mean \pm standard deviation $( \pm S D)$. Student's t-test were used to test for difference between two independent means or proportions, and $p \leq$ 0,05 was considered statistical significant.

\section{Ethical approval}

Ethical approval was obtained from the Regional Scientific Committee and patients provided written informed consent.

\section{Results}

Seventeen adult patients with a confirmed CF diagnosis who were registered at the Copenhagen $\mathrm{CF}$ centre and who fulfilled inclusion and exclusion criteria were invited to participate in the study. Three declined due to private reasons, one dropped out due to regular feverish episodes caused by home fungi. The demographics for the remaining thirteen patients are shown in table 1 .

Both the mean annual change rate for annual $\mathrm{FEV}_{1}$ \%pred and $\mathrm{FVC}$ \%pred changed from decline to improvement after the intervention, although non-significantly (Figure 1$)$. Mean FRC was $3,1( \pm 1,3)$ liter at study start and $3,2( \pm 0,9)$ liter at the end. The mean LCI was initially $19,0( \pm 3,7)$ units and improved to $17,3( \pm 2,8)$ at the end, n.s. Some of those who had reduced (improved) LCI showed increased FRC, others vice versa. The mean chest mobility at the start of the intervention was $5,7( \pm 1,2) \mathrm{cm}$ and improved to $7,2( \pm 1,3) \mathrm{cm}$ at the end of the study, $\mathrm{p}=0,004$. Neither FRC, LCI nor chest mobility had been measured during the control period.

The mean PWC \%pred at start of intervention was $83,2( \pm 10,9)$ and improved to $88,1( \pm 13,9), \mathrm{p}=0,04$. The mean $\mathrm{SpO} 2$ increased from $95,9( \pm 2,3)$, to $97,0( \pm 2,3)$ at the improved PWC, $\mathrm{p}=0,02$. The PWC had not been measured routinely prior to the intervention. Mean BMI maintained $25( \pm 0,4)$ during control and intervention period.

The mean hours of physical activity performed during a normal 24 hour-day was at the start of the study $4,7( \pm 2,4)$ and had changed to $5,3( \pm 1,7)$ after the intervention, n.s. Seven of the 13 were performing high intense physical activity every day at the start, while 11 of the 13

Table 1. Baseline data at the start of the intervention. Expressed as number of patients if nothing else is told

\begin{tabular}{|l|l|}
\hline Variable & \\
\hline Number & 13 \\
\hline M/F & $6 / 7$ \\
\hline Age, years: & \\
\hline mean $( \pm \mathrm{SD})$ & $43( \pm 6.7)$ \\
\hline median $($ range $)$ & $39(32-51)$ \\
\hline FEV1 \%pred, mean $( \pm \mathrm{SD})$ & $59( \pm 11.3)$ \\
\hline FVC $\%$ pred, mean $( \pm \mathrm{SD})$ & $80( \pm 12.1)$ \\
\hline BMI $($ weight/length $)$, mean $( \pm \mathrm{SD})$ & $25( \pm 0.4)$ \\
\hline Genetics: & \\
\hline$\Delta \mathrm{F} 508 / \Delta \mathrm{F} 508$ & 9 \\
\hline$\Delta \mathrm{F} 508 /$ other & 4 \\
\hline Sputum colonisation: & \\
\hline No chronic colonisation & 1 \\
\hline Pseudomonas aeruginosa, chronic & 10 \\
\hline Burkholderia cepacia, chronic & 3 \\
\hline Nontubercolous mycobacteria, chronic & 1 \\
\hline Achromobacter, chronic & 1 \\
\hline
\end{tabular}


did so at the end of the study. Quality of life was measured with the help of four domains from the CFQ-R for adults [23]. The difference for the four domains between start and end of the intervention were all positive, but not significant (Table 2). The respiratory domain showed the highest improvement.

\section{Discussion}

It is known that one AC technique is not superior to CF patients in general but may be for the single individual, which should induce us to individualize the treatment [4-6]. Physical capacity, social situation, character and daily condition differ and are reasons for us to personalize the treatment together with the single patient continuously. We considered all AC therapy during the intervention to be founded on the same respiratory physiology strategy, a "treatment cycle", while the physiotherapy components in the individual treatment programmes are based on A) the physiologic effects achieved from the alternative treatment techniques tested, and B) on patient preference. Since physiological and social conditions change the technique(s) utilized may differ over time, but also from one day to another, or from morning to evening, again considering efficiency and preference. We are convinced that adherence is improved in patients who are educated in the treatment, who have had the opportunity to try different techniques and are provided with choice, as reported in a study by McIlwaine (2013) with $94 \%$ adherence [25]. Adherence was not a parameter in our study since declining adherence functioned as

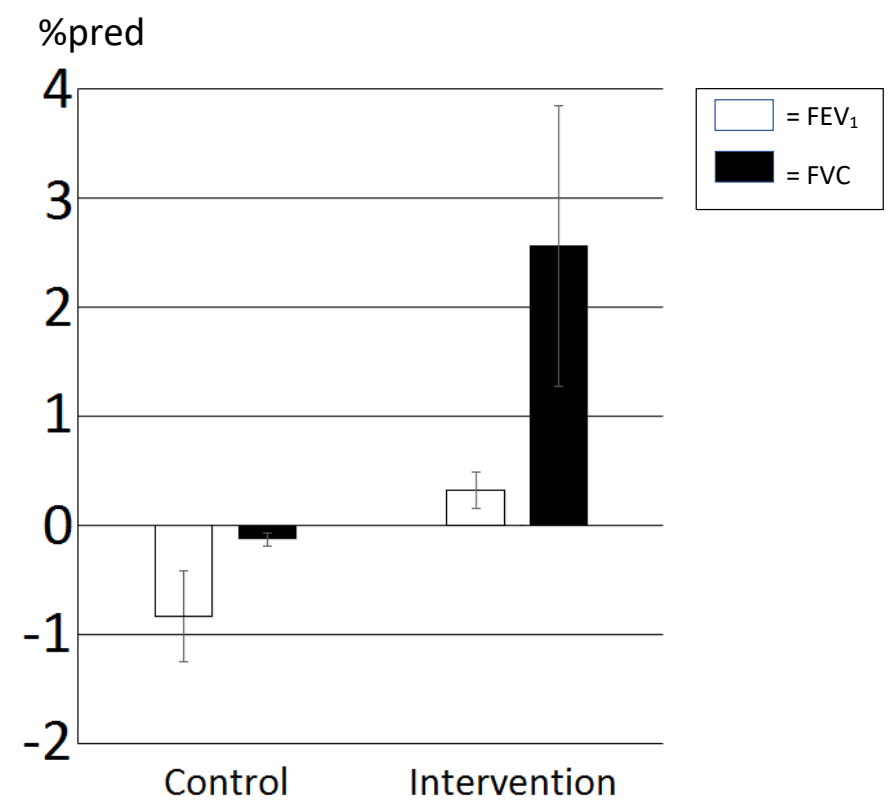

Figure 1. The mean annual lung function change rate \pm SD the immediate control years prior to and then after the intervention year

White $=$ Forced Expiratory Volume in one second $\left(\mathrm{FEV}_{1}\right)$ and black $=$ Forced Vital Capacity (FVC).

\begin{tabular}{|c|c|c|}
\hline Domain & At start & At end \\
\hline Physical & $81.4( \pm 12.1)$ & $83.3( \pm 11.9)$ \\
\hline Emotional & $80.0( \pm 19.6)$ & $83.1( \pm 13.5)$ \\
\hline Health perception & $55.6( \pm 26.0)$ & $57.3( \pm 19.8)$ \\
\hline Respiratory & $68.8( \pm 15.0)$ & $73.3( \pm 13.1)$ \\
\hline
\end{tabular}

Table 2. Quality of life*, mean \pm SD at the start and at the end of the intervention, n.s *The Danish version of the revised CF quality of life questionnaire in adolescents and adults, CFQ-R domains, scale $0-100$ [23]. an incentive for optimising/modifying/changing technique before it became a problem.

Although participants in our study had relatively high fitness levels at the start of the intervention, they still establish that physical exercise, if included as a part of the basic treatment in a longer term may result in increased PWC. Their improved SpO2 at the increased PWC level suggests an even further improvement of the ventilation capacity. Although low during the control period, the annual mean decline rate of $\mathrm{FEV}_{1}$ \%pred changed to positive by a year of individualized and continuously optimized physiotherapy, including physical exercise. In a longer follow-up we do not know what the result would be. The change of rate cannot be expected to follow this pattern but perhaps be more stable than is the $\mathrm{FEV}_{1}$ \%pred decline rate for deltaF508 homozygous patients that is $-1,52$ and for deltaF508 heterozygous $-1,35$, recently reported by De Boeck (2017) from the ECFS registry [26]

\section{Benefits from physical exercise}

Physical exercise resulting in slower rate of lung function decline is well known and many patients report improved AC after physical exercise sessions $[6,10-13,27,28]$. One theory is clogged or collapsed airways may be recruited by lung tissue interdependence, due to temporarily increased lung volumes such as tidal volume (TV) and/ or FRC (depending on lung condition) during the physical exercise $[29,30]$. Another theory is that during exercise in CF, the temporarily reduced sodium and water re-absorption from the airway mucosa enables mucociliary function [31]. A combination of both may make the mucus dislodge efficiently from airway walls. In conformity with that, many patients utilize physical exercise a time efficient alternative AC technique, when combining it with the forced expiration technique [6,7]. Another important factor for the result may be adherence, which in a previous study by White (2007) has been reported $91 \%$ to physical exercise [28]. As been reported previously we found a bigger effect on FVC than on FEV 1 pred [10-12]. Maybe this is due to that if pulmonary hyperinflation has not been adequately treated the chest wall becomes stiff and hampers deep expirations, which hinders AC therapy at low lung volume. Improving expiratory mobility in a stiff chest is possible and may be difficult and time-consuming, sustaining mobility is therefore of interest in basic CF care [32]. Utilizing an AC technique mobilizing mucus at an as low Residual Volume (RV) as possible (step 2 in the treatment cycle) may simultaneously maintain or even improve chest mobility, which may have been confirmed in this study. However, each AC session is being finished with deep inspirations to avoid micro-atelectasis.

Out of musculoskeletal aspects osteoporosis is a growing problem, vertebral fractures are common and thoracic kyphosis have been reported in about $50 \%$ of adult CF populations [27,32]. To an increasing extent physical exercise as prevention to musculoskeletal complications is included in the basic CF care, and part of the exercise is recommended to be weight-bearing to sustain bone mass density (BMD) $[3,6,27,32]$. BMD was not collected in this study, but endurance training was always partly carried out weight-bearing, considering future consequences. BMD ought to be included in the outcomes in future studies. Similarly, the increased demands on ventilation during the physical exercise may strengthen respiratory muscles, thereby it may not be necessary to utilize specific time for inspiratory muscle training [33]. However, inspiratory muscle strength was not collected in this study, but the population could be interesting to utilize as comparing in a study reaching across different centres. Just as previously reported, our experience is that good posture reduces the risk for musculoskeletal dysfunction [32]. Therefore, chest wall and 
auxiliary muscle mobility exercises and muscle strengthening exercises embracing postural muscles were included to maintain or improve these functions, striving for avoiding dysfunction and back pain, and preserving a "healthy look".

\section{Lung clearance index and open airways}

Obstructive small airways contribute little to airway resistance. Therefore, LCI evaluating effects in the small airways and on ventilation is more interesting than forced expiratory lung tests that show lack of sensitivity to early disease [34]. However, LCI is less informative when there are airways not reachable for measuring, such as collapsed airways and where air is trapped by e.g. secretion plugs. These are situations that change with recruited lung volumes and removed plugs, where the re-opened airways may be obstructive and available ventilation is paradoxically aggravating the LCI [35]. Such results in this small study may be due to coincidence, but if not, it supports that the separate effects on FRC and LCI from physiotherapy in inhomogeneous ventilation are complex and not predictable [35].

\section{Quality of life}

Although quality of life was high among the adult CF patients at the start of the intervention, it increased further. The four CFQ-R domains were utilized since they expected to contain the most information about physical status $[23,36]$. We believe that increased intensity in daily physical activity can be a reason to the improved quality of life, especially when the respiratory domain is the increased, as reported. Utilizing PAS can be questioned since it has not yet been validated in $\mathrm{CF}$, but it contains much alternative flexibility and a functional aspect measuring intensity that we regard adequate [24]. We considered it an easy tool with a close cultural connection, therefore suitable to use.

\section{Frequent contact}

Patients invited to participate were accustomed to coming for scheduled check-up to the hospital's CF out-patient-clinic once every 4-6th week, but not to the physiotherapist prior to the study. By routine the physiotherapist used to be involved in connection to annual control, and in between if a physician or nurse considered there was a need or if patients asked for it themselves.

While enrolled in this study, patients met a physiotherapist at each of their "scheduled" routine visit, supporting importance of regular assessment $[5,10]$. Adequate functional aspects concerning the content of the physiotherapy were defined individually as the base for evaluating effect and adherence with the treatment. The meetings were utilized for individual education, repeating or catching up with previous discussion and the current treatment techniques were evaluated, quality optimized or techniques exchanged. This study included weekly contacts, usually by telephone, aiming at supporting treatment motivation. The frequent contacts required a lot of time for both patient and physiotherapist, but our experience says it is of great value for education and motivation while optimizing/modifying/changing a treatment programme. Maintained increase of physical activity in habitual everyday life might represent a change in life style that may preserve longer lasting benefits from the improved PWC, SpO2 and lung function [6-7,10-13,17].

\section{Conclusion}

This one-year study is of the first of its kind that made it possible to continuously utilize all parts of modern physiotherapy individually the best in clinical work, as prerequisites change. Although small and in absence of a control group, this long-term study shows positive effects on PWC, lung function, intensity of daily physical activity and that quality of life may be improved, if physiotherapy is frequently evaluated and re-designed in collaboration with the patient. The requirements for good long-term results seem to be continuous updating and educating about optimal use of physiotherapy, functioning as a base for the physiotherapist and single patient to jointly find sufficient but not too extensive treatment that fit into everyday life. Considering the number of CF patients may grow faster than team resources, there is need for unsupervised, but individualized and frequently optimized physiotherapy as an important part of basic care to counteract pulmonary disease progress. Further and longer studies on effects from early cooperation between physiotherapist and patient in all ages are needed.

\section{Acknowledgement}

\section{Funding information}

This research received grant from the Obelske Family Fund, Denmark.

\section{Competing interest}

The authors declare that they have no competing interests.

\section{References}

1. Boucher RC (2007) Cystic fibrosis: a disease of vulnerability to airway surface dehydration. Trends Mol Med 13: 231-240. [Crossref]

2. Castellani C, Duff AJA, Bell SC, Heijerman HGM, Munck A, et al. (2018) ECFS best practice guidelines: the 2018 revision. J Cyst Fibros 17: 153-178. [Crossref]

3. International Physiotherapy Group for Cystic Fibrosis (IPG/CF) (2009) Physiotherapy for people with Cystic Fibrosis: from infant to adult. (4th edn). [Available at: https:// www.ecfs.eu/ipg_cf/booklet]

4. Mcllwaine MP, Lee Son NM, Richmond ML (2014) Physiotherapy and cystic fibrosis what is the evidence base? Curr Opin Pulm Med 20: 613-617. [Crossref]

5. Rand S, Hill L, Prasad SA (2013) Physiotherapy in cystic fibrosis: optimising techniques to improve outcomes. Paediatr Respir Rev 14: 263-269. [Crossref]

6. Dennersten U, Lannefors L, Höglund P, Hellberg K, Johansson H, et al. (2009) Lung function in the aging Swedish cystic fibrosis population. Respir Med 103: 1076-1082. [Crossref]

7. Andréasson B, Jonson B, Kornfält R, Nordmark E, Sandström S (1987) Long-term effects of physical exercise on working capacity and pulmonary function in cystic fibrosis. Acta Paediatr Scand 76: 70-75. [Crossref]

8. Wang YB, Watts AB, Peters JI, Williams RO (2014) The impact of pulmonary diseases on the fate of inhaled medicines--a review. Int J Pharm 461: 112-128. [Crossref]

9. Brand P, Meyer T, Häussermann S, Schulte M, Scheuch G, et al. (2005) Optimum peripheral drug deposition in patients with cystic fibrosis. J Aerosol Med 18: 45-54. [Crossref]

10. Moorcroft AJ, Dodd ME, Morris J, Webb AK (2004) Individualised unsupervised exercise training in adults with cystic fibrosis: a 1 year randomised controlled trial. Thorax 59: 1074-1080. [Crossref]

11. Hebestreit H, Kieser S, Junge S, Ballmann M, Hebestreit A, et al. (2010) Long-term effects of a partially supervised conditioning programme in cystic fibrosis. Eur Respir J 35: 578-583. [Crossref]

12. Schneiderman-Walker J, Pollock SL, Corey M, Wilkes DD, Canny GJ, et al. (2000) A randomized controlled trial of a 3 -year home exercise program in cystic fibrosis. $J$ Pediatr 136: 304-310. [Crossref]

13. Schneiderman JE, Wilkes DL, Atenafu EG, Nguyen T, Wells GD et al. (2014) Longitudinal relationship between physical activity and lung health in patients with cystic fibrosis. Eur Respir J 43: 817-823. [Crossref]

14. Hebestreit H, Arets HG, Aurora P, Boas S, Cerny F et al. (2015) Statement on Exercise Testing in Cystic Fibrosis. Respiration 90: 332-351. [Crossref]

15. Orenstein DM, Franklin BA, Doershuk CF, Hellerstein HK, Germann KJ, et al. (1981) Exercise conditioning and cardiopulmonary fitness in cystic fibrosis. The effects of a three-month supervised running program. Chest 80: 392-398. [Crossref] 
16. Kriemler S, Kieser S, Junge S, Ballmann M, Hebestreit A, et al. (2013) Effect of supervised training on $\mathrm{FEV}_{1}$ in cystic fibrosis: a randomised controlled trial. $J$ Cyst Fibros 12: 714-720. [Crossref]

17. Cox NS, Alison JA, Button BM, Wilson JW, Morton JM, et al. (2016) Physical activity participation by adults with cystic fibrosis: An observational study. Respirology 21 : 511-518. [Crossref]

18. Quanjer PH, Stanojevic S, Cole TJ, Baur X, Hall GL, et al. (2012) Multi-ethnic reference values for spirometry for the 3-95-yr age range: the global lung function 2012 equations. Eur Respir J 40: 1324-1343. [Crossref]

19. Robinson PD, Goldman MD, Gustafsson PM (2009) Inert gas washout: theoretical background and clinical utility in respiratory disease. Respiration 78: 339-355. [Crossref]

20. Godfrey S. Exercise testing in children. London (1974) W.B. Saunders Company Ltd,1-168.

21. Nordenfelt I, Adolfsson L, Nilsson JE, Olsson S (1985) Reference values for exercise tests with continuous increase in load. Clin Physiol 5: 161-172. [Crossref]

22. Borg GA (1982) Psychophysical bases of perceived exertion. Med Sci Sports Exerc 14 377-381. [Crossref]

23. Bregnballe V, Thastum M, Lund LD, Hansen CR, Preissler T, et al. (2008) Validation of the Danish version of the revised cystic fibrosis quality of life questionnaire in adolescents and adults (CFQ-R14+). J Cyst Fibros 7: 531-536. [Crossref]

24. Aadahl M, Jørgensen T (2003) Validation of a new self-report instrument for measuring physical activity. Med Sci Sports Exerc 35: 1196-1202. [Crossref]

25. McIlwaine MP, Alarie N, Davidson G, Lands LC, Ratjen F, et al. (2013) Long-term multicentre randomised controlled study of high frequency chest wall oscillation versus positive expiratory pressure mask in cystic fibrosis. Thorax 68:746-751. [Crossref]

26. De Boeck K, Zolin A (2017) Year to year change in FEV, in patients with cystic fibrosis and different mutation classes. J Cyst Fibros 16: 239-245. [Crossref]
27. Tejero Garcia S, Giráldez Sánchez MA, Cejudo P, Quintana Gallego E, Dapena J et al. (2011) Bone health, daily physical activity, and exercise tolerance in patients with cystic fibrosis. Chest 140: 475-481. [Crossref]

28. White D, Stiller K, Haensel N (2007) Adherence of adult cystic fibrosis patients with airway clearance and exercise regimens. J Cyst Fibros 6: 163-170. [Crossref]

29. Regnis JA, Alison JA, Henke KG, Donnelly PM, Bye PT (1991) Changes in endexpiratory lung volume during exercise in cystic fibrosis relate to severity of lung disease. Am Rev Respir Dis 144: 507-512. [Crossref]

30. Aliverti A (2008) Lung and chest wall mechanics during exercise: effects of expiratory flow limitation. Respir Physiol Neurobiol 163: 90-99. [Crossref]

31. Hebestreit A, Kersting U, Basler B, Jeschke R, Hebestreit H (2001) Exercise inhibits epithelial sodium channels in patients with cystic fibrosis. Am J Respir Crit Care Med 164: 443-446. [Crossref]

32. Lima T, Guimaraes F, Ferreira A, Penafortes J, Almeida V, et al. (2014) Correlation between posture, balance control and peripheral muscle function in adults with cystic fibrosis. Physiother Theory Pract 30: 79-84. [Crossref]

33. Dassios T, Katelari A, Doudounakis S, Dimtriou G (2013) Aerobic exercise and respiratory muscle strength in patients with cystic fibrosis. Respir Med 107: 684-690. [Crossref]

34. Ratjen F (2012) Cystic fibrosis: the role of the small airways. J Aerosol Med Pulm Drug Deliv 25: 261-264. [Crossref]

35. Pfegler A, Steinbacher M, Schwantzer G, Weinhandl E, Wagner M, et al. (2015) Shortterm effects of physiotherapy on ventilation inhomogeneity in cystic fibrosis patients with a wide range of lung disease severity. J Cyst Fibros 14: 627-631.[Crossref]

36. Hebestreit H, Schmid K, Kieser S, Junge S, Ballmann M, et al. (2014) Quality of life is associated with physical activity and fitness in cystic fibrosis. BMC Pulm Med 14: 26. [Crossref]

Copyright: (C2018 Lannefors L. This is an open-access article distributed under the terms of the Creative Commons Attribution License, which permits unrestricted use, distribution, and reproduction in any medium, provided the original author and source are credited. 